\title{
Hybrid active and passive control of vibratory power flow in flexible isolation system
}

\author{
Y.P. Xiong ${ }^{\mathrm{a}, \mathrm{b}}$, J.T. Xing ${ }^{\mathrm{b}}$, W.G. Price ${ }^{\mathrm{b}}$ and \\ X.P. Wang ${ }^{\mathrm{a}}$ \\ ${ }^{a}$ Institute of Engineering Mechanics, Shandong \\ University of Technology, Jinan, 250061, P.R. China \\ E-mail: xiongyp@dms.sdut.edu.cn, \\ yeping@ship.soton.ac.uk \\ ${ }^{\mathrm{b}}$ School of Engineering Sciences, Ship Science, \\ University of Southampton, Southampton SO17 1BJ, \\ UK \\ E-mail: j.t.xing@ship.soton.ac.uk, \\ w.g.price@ship.soton.ac.uk
}

\section{Received 2 April 1999}

Revised 15 March 2000

\begin{abstract}
A hybrid active and passive vibration control strategy is developed to reduce the total power flows from machines, subject to multiple excitations, to supporting flexible structures. The dynamic interactions between machines, controllers, and receiving structures are studied. A force feedback control process governed by a proportional control law is adopted to produce active control forces to cancel the transmitted forces in the mounts. Computational simulations of a simple and a multiple dimensional hybrid vibration isolation system are performed to study the force transmissibility and the total power flows from vibration sources through active and passive isolators to the supporting structures. The investigation focuses on the effects of a hybrid control approach to the reduction of power flow transmissions and the influence of the dynamic characteristics of the control on power flow spectra. The hybrid control mechanism is synthesised from the power flow analysis. Conclusions and control strategies, well supported by numerical simulations, are deduced providing very useful guidelines for hybrid vibration isolation design.
\end{abstract}

\section{Introduction}

It is well known that passive vibration isolation is a proven approach to reduce vibration transmission between vibration sources and receiving structures $[4,6$, 14]. However, conflicting requirements for conven- tional passive isolation are often encountered when a heavier machine is mounted on a flexible supporting structure. For example, the required stiffness of the mounts to support the machine may be far greater than required for them to act as possible vibration isolators, thus limiting their isolation capability. Moreover, when the receiving structure and/or source are flexible, the dynamic design of the isolator system becomes an interesting issue because of the complex dynamic interaction between the substructures of the isolation system. The effects of vibration isolator properties, and the influence of structural dynamics of flexible raft and supporting foundation on isolator performance, were discussed by Xiong et al. $[15,16]$ to illustrate the importance of the dynamic interaction between isolators, raft and resonances in the flexible foundation at higher frequencies.

A recent innovation in vibration isolation technology is to use optimal, adaptive and active control techniques to improve the performance of conventional isolation systems. Franchek et al. [3] achieved robust attenuation of a harmonic excitation through an adaptive passive control strategy. Scribner et al. [11] focused on the study of active narrow-band control of machinery noise from resonant substructures. Improvement of isolation effectiveness via active control strategies was undertaken by Jenkins et al. [7]. Although active isolation systems can achieve better performance than their passive counterparts, they are typically implemented in combination with a passive isolator to take advantage of their respective capbilities. Therefore, investigations of hybrid active and passive vibration isolation systems have received much attention. Hybrid control strategies have been studied to exploit their potential to increase the overall reliability and efficiency of controlled systems. Shing et al. [13] and Kawatani et al. [8] examined optimal control methods for hybrid controller designs. Grigoriadis et al. [5] presented an optimal controller based on passive and active control methods to achieve a numerical solution of a quadratic programming problem. Leo and Inman [9] developed a quadratic programming algorithm to study the design 
trade-offs of active-passive vibration isolation systems in which the optimal control problem was treated as a quadratic optimization with linear constraints to guarantee the global optimal solution of the hybrid isolation system. Pare and How [10] proposed a hybrid feedforward and feedback controller design approach for structural vibration control and gave a simple structural isolation example to demonstrate the benefit of the optimal hybrid controller to improve simultaneously isolation performance together with a reduction in closed loop control bandwidth. Sener and Utku [12] studied a hybrid base isolation system by using passive base isolation devices and strain inserting actuators. Xiong and Song [17] studied an optimal control method applied to power flow to reduce vibration energy transmissions in multiple dimensional isolation systems.

The main focus of the present paper is to investigate a hybrid active and passive energy control strategy from the viewpoint of power flow analysis examining the effects induced by a distributed receiver and by a multiple hybrid mounting system. The proposed mathematical model describes the dynamic interactions between machine, passive/active controllers and the dynamics of the supporting structure in a multi-dimensional flexible coupled system subject to multiple excitations. In the examined multiple hybrid mounting system, each active actuator is placed in parallel with a passive mount in the load path of the isolation system. The feedback control mechanism of power flow in this flexible coupled dynamic system is investigated. In particular, the investigation examines the effects of hybrid active and passive mounts on the reduction of power flow transmission. Strategies to control the net power flow are proposed so as to achieve good isolation of the machinery and to control base vibrations.

\section{A simplified hybrid active and passive isolation system}

To understand clearly the control mechanism of the hybrid active and passive isolation system, a simplified mathematical model with one degree of freedom is first studied, for example, a system idealized by a machine mounted on a flexible foundation floor.

\subsection{Dynamic description of the simple hybrid control system}

Figure 1 illustrates schematically a simple hybrid active and passive isolation system with one degree of freedom, which represents a typical engineering configuration of a parallel type isolation design. A machine A of mass $m$, excited by a harmonic force $f=f_{0} e^{j \omega t}$, is mounted on a supporting beam-like structure $\mathrm{C}$ of length $L$ through a mounting system B consisting of a passive isolator and an active actuator. In this figure $k$ and $c$ represent the spring stiffness and the damping coefficient of the passive isolator, respectively. The centre of gravity of the machine is located at the point $G$ which corresponds to the mounting point on the foundation $\mathrm{C}$ denoted by a position parameter $\mu(0<\mu<1)$. The case $\mu=0.5$ denotes a symmetric configuration of the system. The origins of the coordinates $x_{1}$ and $x_{2}$ are located at the points $G$ and $O$, respectively, of the system in its static equilibrium state as shown in Fig. 1. The active actuator is placed in parallel with the passive isolator in the load path. The axial control force, exerted simultaneously on both the machine and the supporting flexible structure, is driven by a suitable feedback control process to control, in an active manner, the vibration transmission. It is assumed that the internal structural damping of the beam-like foundation $\mathrm{C}$ is represented by $E^{*}=E(1+j \delta)$, where $\delta$ is the damping factor of the beam material and $E$ its Young's modulus.

The dynamics of the hybrid active and passive isolation system are described by the following equations. That is, the motion of the mass $m$ is governed by

$$
m \ddot{x}_{1}+c\left(\dot{x}_{1}-\dot{x}_{2}\right)+k\left(x_{1}-x_{2}\right)=f-f_{c} .
$$

The resultant force $f_{t}$ transmitted through the passive and active mounting system $B$ to the foundation $C$ is represented as (2)

$$
f_{t}=f_{c}+f_{p}=f_{c}+\left(x_{1}-x_{2}\right)(k+j c \omega),
$$

where $f_{p}$ and $f_{c}$ are the passive and the active control forces acting through the mounting axis, respectively.

The dynamic equation governing the flexible foundation, formulated in terms of a mobility function $M$ at the mounting point $O$, is expressed as

$$
\begin{aligned}
x_{2}=M f_{t}= & \frac{f_{t}}{m_{b}} \sum_{k=1}^{\infty} \\
& \frac{\left(\beta^{*}\right)^{4}}{\left(\beta^{*}\right)^{4}-1} \frac{\varphi_{k}^{2}(\mu L)}{\omega_{k}^{2}(1+j \delta)},
\end{aligned}
$$

where $\left(\beta^{*}\right)^{4}=(1+j \delta)\left(\omega_{k} / \omega\right)^{2}$, and $\varphi_{k}$ represent the $k$-th natural frequency and the corresponding normal mode function of the clamped-clamped beam, respectively. $m_{b}$ denotes the total mass of this beam.

An application of a proportional force feedback control law with gain $K_{c}$ gives the control force as

$$
f_{c}=-K_{c} f_{t} \text {. }
$$




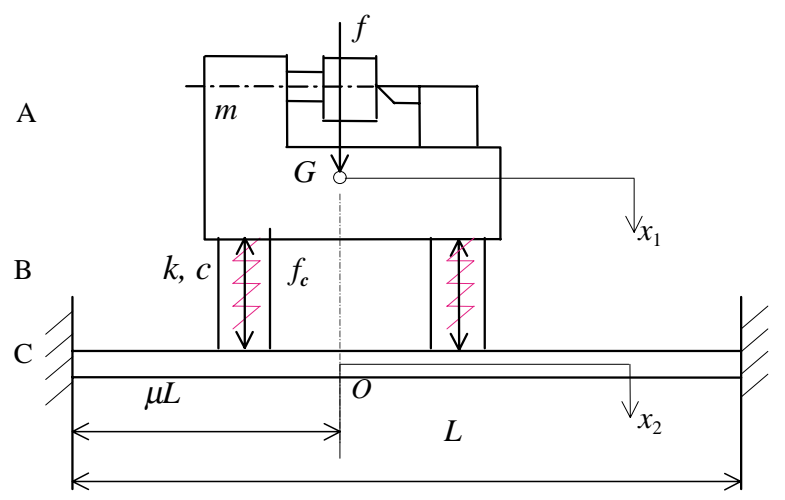

Fig. 1. Demonstration of a simple hybrid isolation system with active actuator in parallel with passive isolator.

\subsection{The force transmissibility function}

It follows from equations (1)-(4) that the force transmissibility function $T$ of the hybrid active and passive isolation system is of the form

$$
\begin{aligned}
T & =\frac{f_{t}}{f} \\
& =\frac{1+2 j \Omega \xi}{\left(1+K_{c}\right) \Omega^{2}-\left(1-\omega^{2} m M\right)(1+2 j \Omega \xi)},
\end{aligned}
$$

where $\Omega=\omega / \omega_{0}, \omega_{0}=\sqrt{k / m}, \xi=c /(2 \sqrt{k m})$. The ratio of the control force to the excitation force is given by

$$
\frac{f_{c}}{f}=-K_{c} T
$$

It can be seen from equation (5) that the magnitude of the force transmissibility function $T$ tends to zero as the feedback gain $K_{c}$ tends to an infinite value. This limit case indicates that there is no resultant force transmitted to the foundation, with is independence on excitation frequency as $K_{c} \rightarrow \infty$.

The variations of the force transmissibility $T$ to changes of the feedback gain $K_{c}$ are shown in Fig. 2, where the mass ratio $\gamma=\mathrm{m} / \mathrm{m}_{b}$ and $\mu=0.5$. The case $K_{c}=0$ indicates the uncontrolled passive isolation system. There are two peaks occurred in this curve, the first pronounced one is due to the resonance of the isolated system and the second one is corresponding to the fundamental frequency of the foundation. In the hybrid isolation system, however, the second peak is not observed because of the dynamic stiffness softening effect of the hybrid mount and hence reducing the transmitted force and weakening the dynamic coupling effect between the hybrid mount and the flexible foundation. It can be observed from this figure that the force transmissibility of the hybrid isolation sys- tem is reduced with an increase of the feedback gain $K_{c}$. This is because the active actuator provides an active control force $f_{c}$ to cancel part of the transmitted force of the passive isolator. It is also observed that the resonant frequency of the hybrid active and passive isolation system moves to a lower frequency range as the feedback gain increases $\left(K_{c}>0\right)$. This is due to the control force creating a dynamic stiffness softening effect, but it does not change the static stiffness of the mounts. The hybrid active and passive isolation system can therefore produce a much lower resonant frequency of the system than the passive one only without diminishing the capability of the isolator to support the machine. As a result of this combination, better vibration isolation effectiveness is demonstrated over an extended frequency range.

Figure 3 illustrates the amplitude spectra of the control force represented by equation (6) for the hybrid active and passive control system. It clearly shows that in the lower frequency range, the amplitude of the control force is approximately a constant and it increases as the feedback gain $K_{c}$ increases. However, in the higher frequency range the amplitude of the control force is independent of the feedback. This control force characteristic deduced from equation (6), may provide a starting reference point to examine hybrid isolation systems at higher frequencies where it is hard to generate a larger control force by increasing the feedback gain.

\subsection{Hybrid control of power flow transmission}

The transmitted power through an isolator system, from machine to foundation, is a very important quantity to measure the performance of the isolation system. For the hybrid isolation system studied herein, the total power transmission can be calculated from the 


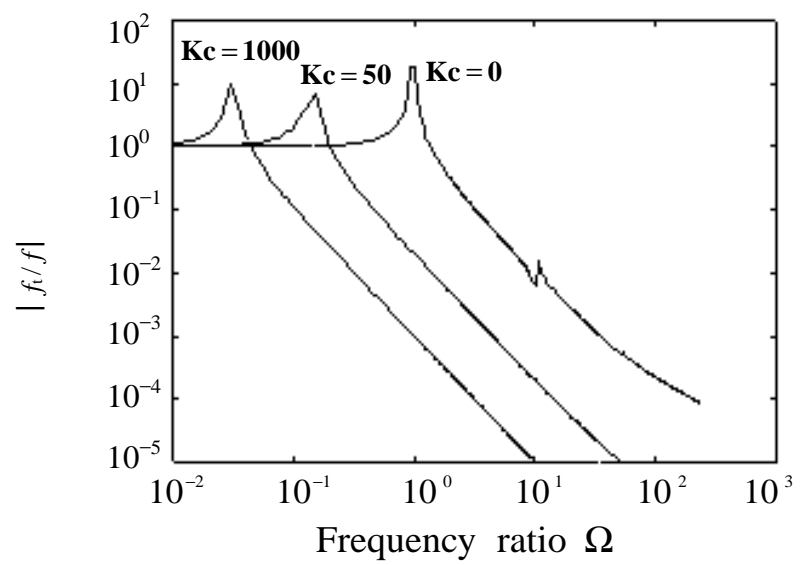

Fig. 2. Comparison of the magnitudes of the force transmitted to the passive and hybrid isolation systems $\left(\mu=0.5, \omega_{1} / \omega_{0}=10\right.$, $\gamma=2, \delta=0.001, \xi=0.01)$.

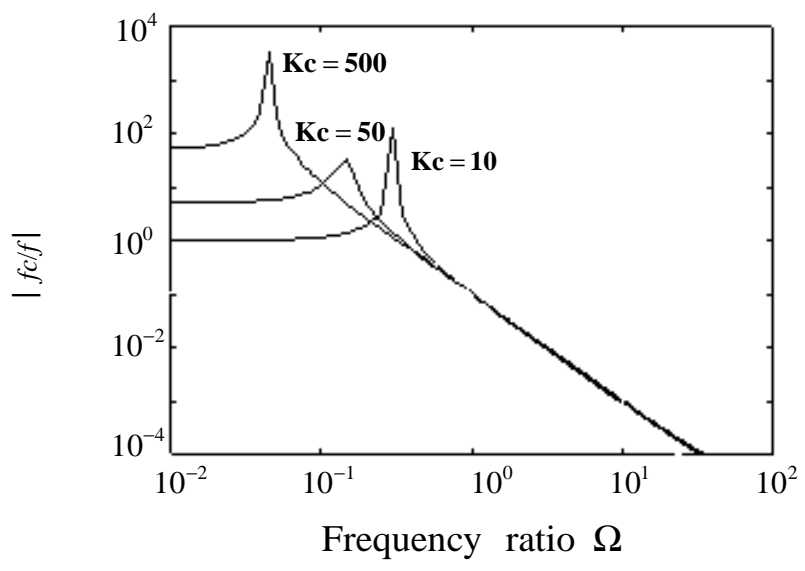

Fig. 3. Control force required for the parallel hybrid mount in the isolation system $\left(\mu=0.5, \omega_{1} / \omega_{0}=10, \gamma=2, \delta=0.001, \xi=0.01\right)$.

expression

$$
P_{t r}=\frac{1}{2} \operatorname{Re}\left\{f_{p}^{*} \nu_{2}\right\}=\frac{1}{2} \operatorname{Re}\left\{j \omega x_{2} f_{t}^{*}\right\}
$$

where the superscript* denotes a complex conjugate.

The substitution of equations (3) and (5) into equation (7) yields

$$
\begin{aligned}
P_{t r} & =\frac{1}{2} \operatorname{Re}\left\{j \omega f^{*} T^{*} M T f\right\} \\
& =\frac{1}{2}|f|^{2} \operatorname{Re}\left\{j \omega T^{*} M T\right\},
\end{aligned}
$$

which further allows definition of the power flow transmission spectrum $Q_{t r}$ in the form

$$
\begin{aligned}
Q_{t r}=\frac{P_{t r}}{|f|^{2}} & =\frac{1}{2} \operatorname{Re}\left\{j \omega T^{*} M T\right\} \\
& =\frac{\omega}{2} \operatorname{Im}\left\{T^{*} M T\right\} .
\end{aligned}
$$

It can be seen from equation (8) that power flow transmission in a hybrid active and passive control system is dependent on the mobility of the foundation and the force transmissibility function $T$ in which the dynamics of the passive isolator and the feedback gain of the collocated active actuator play important roles in reducing the energy transmission.

Figure 4 presents a comparison of the power flow transmission spectra for the passive and hybrid isolation systems. It can be observed that the force feedback control produces a significant reduction in the power flow transmission, especially in the higher frequency domain. A comparison between Fig. 3 and Fig. 4 reveals that a small control force $f_{c}$ in the higher frequency domain can result in a considerable suppression of power flow. 


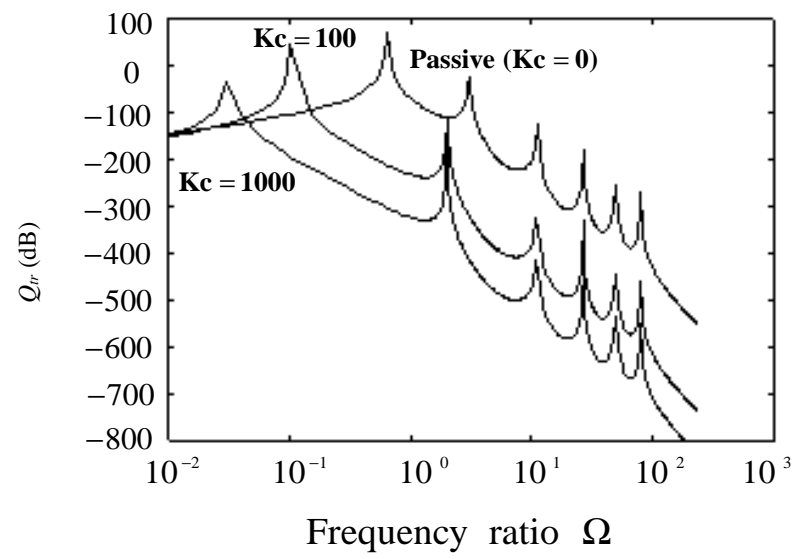

Fig. 4. Comparison of power flow in the passive and hybrid isolation systems $\left(\mu=0.5, \omega_{1} / \omega_{0}=2, \gamma=2, \delta=0.001, \xi=0.01\right)$.

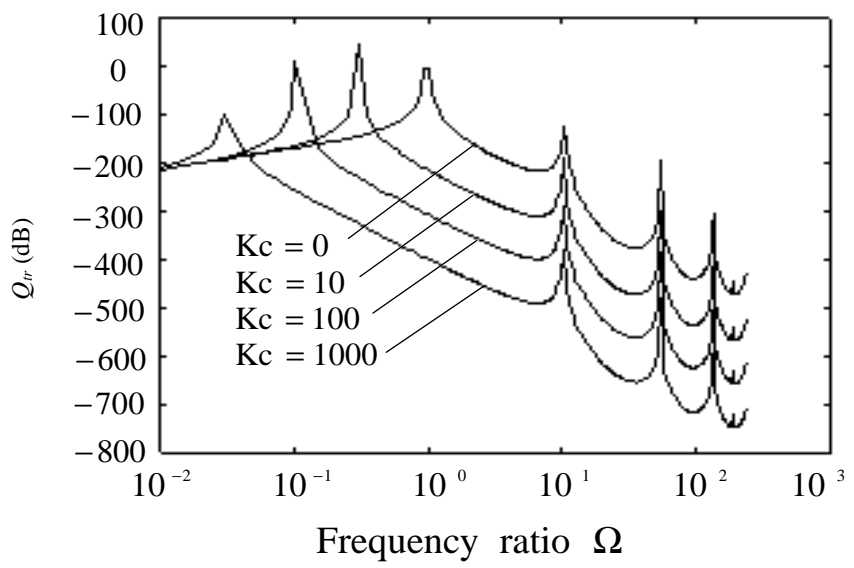

Fig. 5. Variation of power flow spectra to different feedback gain values $\left(\mu=0.5, \omega_{1} / \omega_{0}=10, \gamma=2, \delta=0.001, \xi=0.01\right)$.

It is known that the passive vibration isolation approach is more suitable for the case of machines running at a constant speed and generating vibrations of a specific frequency [6]. However, if this frequency is relatively low the passive approach is ineffective. Figure 5 shows a comparison of power flow spectra for varying feedback gains. It can be seen that the hybrid active and passive isolation system is significantly more effective at lower frequencies than the passive one. As shown in Fig. 2, an increase of the feedback gain results in a move of the resonance frequency to a lower value creating a broader frequency band for effective vibration isolation.

Figure 6 shows a comparison of power flow spectra influenced by the damping factor $\delta$ of the receiving foundation $\mathrm{C}$. It is observed that for the hybrid isolation system an increase of damping causes an increase of power flow transmission in both lower and higher frequency ranges. The effect of the damping factor in the hybrid control system has a similar negative performance as in the passive vibration isolation system at higher frequencies. In the low frequency range, however, for damping $\delta=0.001$, the total power flow transmissions for both the hybrid and passive isolation systems have a similar behavior.

It is also seen from the Figs $4-6$ that the power flow spectra have similar patterns in the high frequency range. This evidence shows that the natural frequencies of the hybrid isolation system, except for the first one, are mainly governed by the dynamic characteristics of the receiver and are not affected by changes of the dynamic stiffness supplied by the active actuator. In contrast, the first natural frequency is affected being associated with the resonance frequency of the isolated 


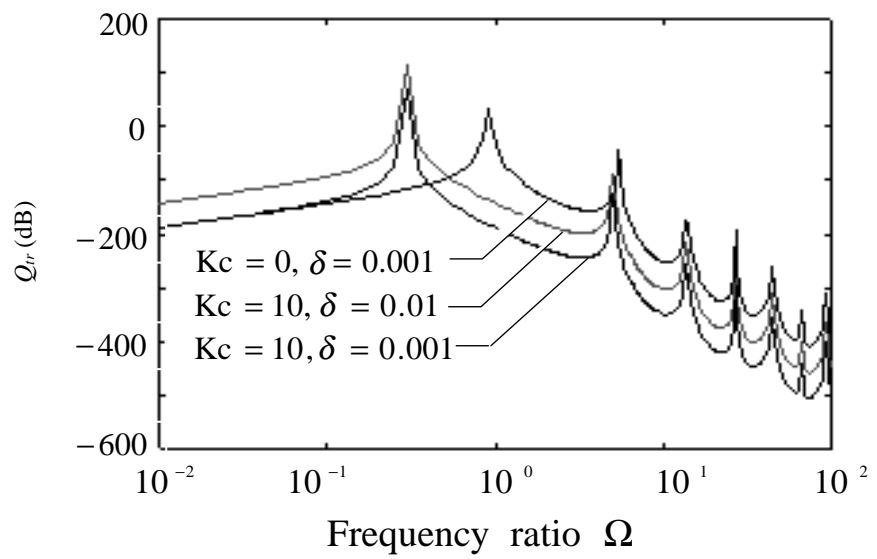

Fig. 6. Power flow control effects influenced by the damping of the receiver $\left(\mu=0.4, \omega_{1} / \omega_{0}=5, \gamma=2, \xi=0.01\right)$.

machine, whereas the other spectra peaks are affected by the resonance behavior of the beam like foundation.

\section{A multi-dimensional hybrid active and passive isolation system}

\subsection{Dynamic description of the hybrid control system}

To extend our study to more practical systems and further explore the behavior of power flow transmission in a hybrid active and passive isolation system, a system with multiple degrees of freedom subject to multi-excitations is studied in this section.

A general hybrid vibration control system with multiple degrees of freedom is shown in Fig. 7(a). The top structure represents a machine to which generalized excitation forces $F_{s}=\left\{F_{s 1}, F_{s 2}, \ldots, F_{s i}, \ldots, F_{s n}\right\}^{T}$ with harmonic frequency $\omega$ are applied. This machine is mounted on a flexible structure through a mounting system consisting of $N$ active and passive mounts. Figure 7(b) illustrates a typical mount, the positive directions of translational and rotational motions and the corresponding force or moment actions. For this system, the passive force transmitted from the vibrating machine through $N$ mounts is denoted by a force vector $F_{p}=\left\{F_{p 1}, F_{p 2}, \ldots, F_{p i}, \ldots, F_{p N}\right\}^{T}$ and $F_{c}=\left\{F_{c 1}, F_{c 2}, \ldots, F_{c i}, \ldots, F_{c N}\right\}^{T}$ represents the active control force vector generated by all actuators. As described previously, the active actuators are placed parallel to each passive mount in the load path.

The motions of the machine and the supporting structure can be expressed by receptance theory [1] as follows

$$
\begin{aligned}
& X_{1}=A_{1} F_{1}+A_{s} F_{s}, \\
& X_{2}=A_{2} F_{2}, \\
& F_{1}=\left[F_{1}^{t}, F_{2}^{t}, \ldots, F_{N}^{t}\right]^{T}, \\
& F_{j}^{t}=\left[F_{x j}^{t}, F_{y j}^{t}, F_{z j}^{t}, M_{x j}^{t}, M_{y j}^{t}, M_{z j}^{t}\right]^{T}, \\
& F_{2}=\left[F_{1}^{b}, F_{2}^{b}, \ldots, F_{N}^{b}\right]^{T}, \\
& F_{j}^{b}=\left[F_{x j}^{b}, F_{y j}^{b}, F_{z j}^{b}, M_{x j}^{b}, M_{y j}^{b}, M_{z j}^{b}\right]^{T}, \\
& (j=1,2, \ldots, N) .
\end{aligned}
$$

where $X_{1}, X_{2}$ are the generalized displacement vectors describing the motions at the top and bottom points of the mounts, respectively; $A_{1}$ and $A_{s}$ represent the point and transfer receptance matrices of the machine, respectively; $A_{2}$ denotes the point receptance matrix of the receiver. They can be determined both theoretically and experimentally.

For this hybrid mounting system, the resultant transmitted force vector $F_{t}$ can be obtained by the summation of the active force $F_{c}$ and passive force $F_{p}$. That is,

$$
\begin{aligned}
& F_{t}=F_{c}+F_{p}, \\
& F_{p}=K^{*}\left(X_{1}-X_{2}\right),
\end{aligned}
$$

where $K^{*}$ is a complex stiffness matrix of order $6 N \times$ $6 N$ for the $N$ isolators.

Application of the proportional force feedback control law gives

$$
F_{c}=-K_{c} F_{t}=-\operatorname{diag}\left[K_{c i}\right] F_{t},
$$

where $K_{c i}(i=1,2, \ldots, 6 N)$ denotes a proportional gain. If the mass of each mount is neglected, it follows that

$$
F_{1}=-F_{t}, \quad F_{2}=F_{t} .
$$




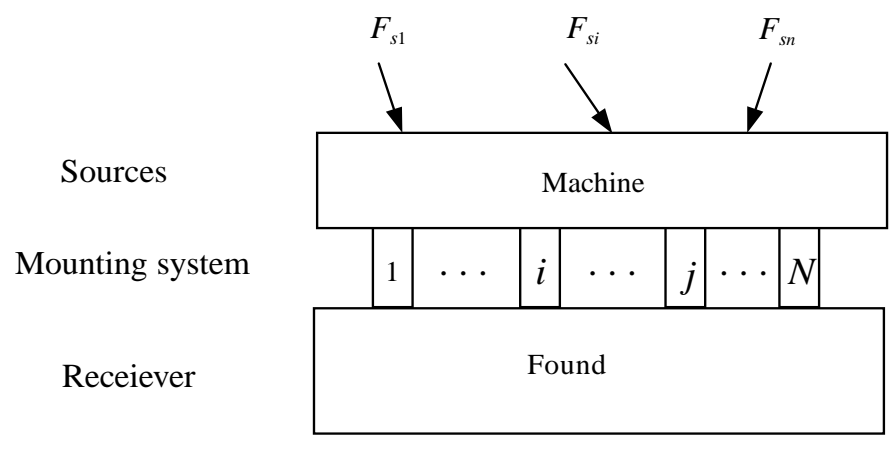

(a) Global system

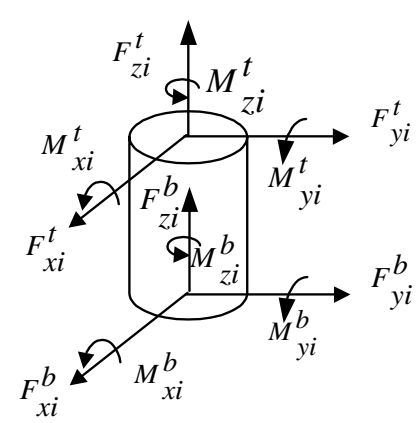

(b) A typical mount

Fig. 7. Sketches of a multi-dimensional hybrid control system model: (a) Global system (b) A typical mount.

\subsection{The force transmissibility matrix}

By using equations (10)-(17), we can derive expressions for the unknown transmitted forces and displacements as follows,

$$
\begin{aligned}
& F_{t}=T(\omega) F_{s}, \\
& X_{2}=A_{2} T(\omega) F_{s}, \\
& X_{1}=A_{s} F_{s}-A_{1} T(\omega) F_{s},
\end{aligned}
$$

where the force transmissibility matrix of the hybrid control system

$$
T(\omega)=\left[I+K_{c}+K^{*}\left(A_{1}+A_{2}\right)\right]^{-1} K^{*} A_{s}
$$

and $I$ represents an unit matrix.

\subsection{Power flow formulation}

The total power transmission from the machine to the foundation of the multiple excitation system can be calculated from the expression

$$
P_{t r}=\frac{\omega}{2} \operatorname{Im}\left\{F_{t}^{H} \cdot X_{2}\right\}
$$

where superscript $H$ refers to a Hermitian operation, i.e., transpose conjugate. The combination of equations (18), (19) and (22) yields

$$
\begin{aligned}
P_{t r}= & \frac{\omega}{2} \operatorname{Im} \\
& \left\{F_{s}^{H} T^{H}(\omega) A_{2} T(\omega) F_{s}\right\} .
\end{aligned}
$$

\subsection{A numerical example of hybrid power flow control}

A more practical hybrid active and passive vibration isolation system with multiple degrees of freedom is examined in this section and is shown in Fig. 8. This system consists of a rigid machine of mass $m$ mounted on a simply supported plate-like floor structure of length $L_{x}$, width $L_{y}$ and thickness $H$ through two identical cylindrical tube-like elastic mounts. The point receptance matrices $A_{1}$ and $A_{s}$ for the rigid machine, as well as the matrix $A_{2}$ for the simply supported plate can be derived theoretically $[1,2]$.

To illustrate the behavior of power flow transmission in the hybrid isolation system, power flow spectra are calculated. The system parameters used for these numerical calculations are described as follows. The machine, subject to a harmonic excitation force vector $F_{s}=F_{s 1}=\left\{f_{1 x} e^{j \omega t}, f_{1 y} e^{j \omega t}, f_{1 z} e^{j \omega t}\right\}^{T}$, is treated as a rigid body of mass $m=32.7 \mathrm{~kg}$ with moments of inertia $I_{x x}=1.4776 \mathrm{~kg} \cdot \mathrm{m}^{2}, I_{y y}=0.0152 \mathrm{~kg} \cdot \mathrm{m}^{2}$ and $I_{z z}=0.0222 \mathrm{~kg} \cdot \mathrm{m}^{2}$. The two identical mounts $(N=2)$ of height $L=0.206 \mathrm{~m}$ are idealized as cylindrical tubes with inner and outer radii $a=0.045 \mathrm{~m}, b=$ $0.051 \mathrm{~m}$, Young's modulus $E_{I}=2.254 \times 10^{7} \mathrm{~N} / \mathrm{m}^{2}$, shear modulus $G_{I}=7.83 \mathrm{~N} / \mathrm{m}^{2}$, Poisson ratio $\left(\lambda_{I}=\right.$ 0.44 and a loss factor $\eta_{i}=0.47(i=1,2)$. To simplify calculations, the same force feedback gain $K_{c}$ is assumed in each mount in all directions. The supporting plate of length $L_{x}=1.8 \mathrm{~m}$, width $L_{y}=1.21 \mathrm{~m}$ and thickness $H=0.005 \mathrm{~m}$ has material properties $E_{p}=2.16 \times 10^{11} \mathrm{~N} / \mathrm{m}^{2}, G_{p}=8 \times 10^{10} \mathrm{~N} / \mathrm{m}^{2}$, $\lambda_{I}=0.3$, and density $\rho=7800 \mathrm{~kg} / \mathrm{m}^{3}$. The first 20 natural modes $(p=20)$ ranging from $2.68 \mathrm{~Hz}$ to $140.68 \mathrm{~Hz}$ are admitted in the power flow calculations. The corresponding modal loss factors are assumed in 


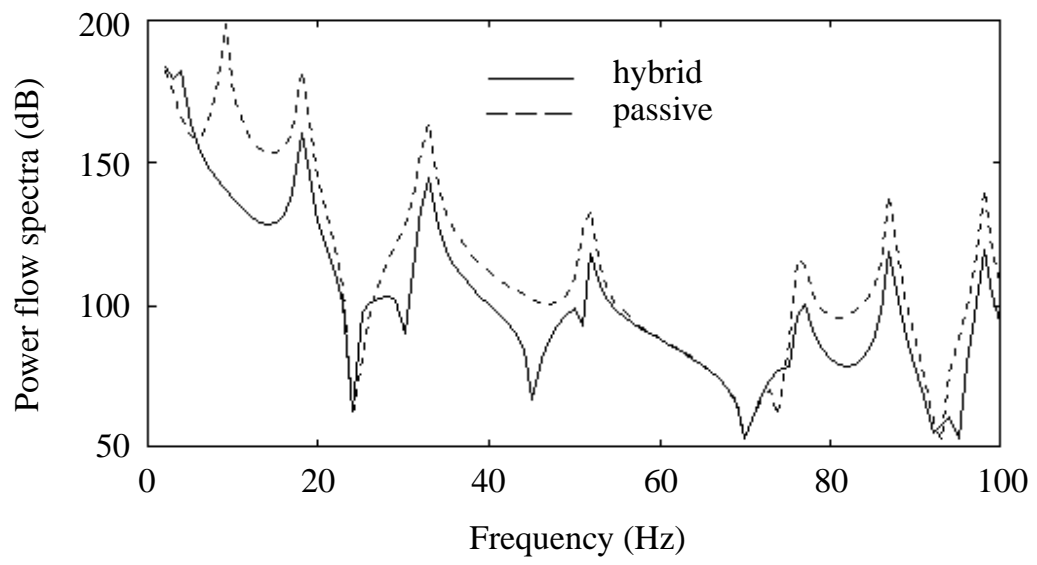

Fig. 8. Hybrid control $\left(K_{c}=5\right)$ effect compared to a passive one $\left(K_{c}=0\right)$.

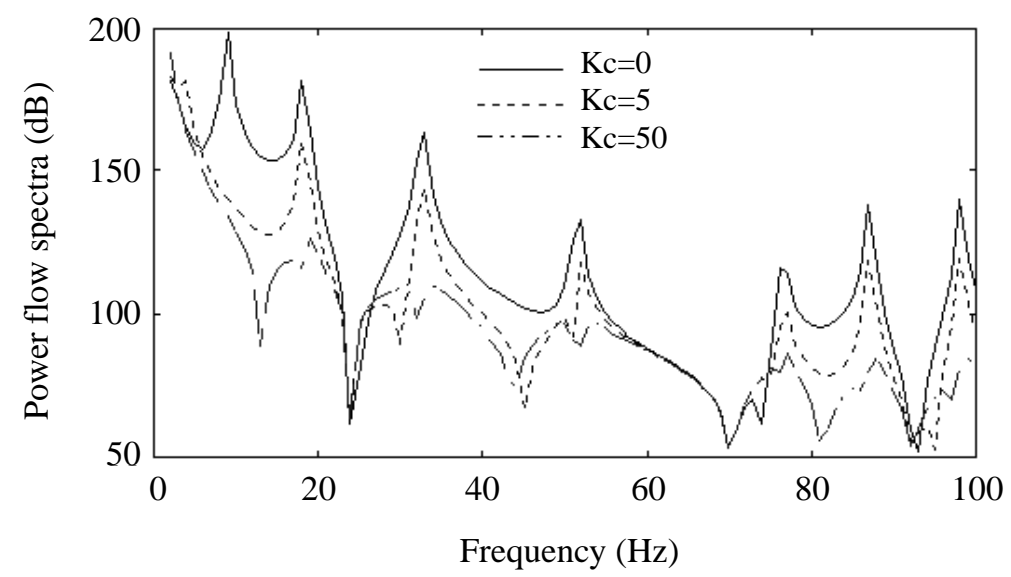

Fig. 9. Hybrid control of power flow for various values of feedback gain.

the form $\delta_{i}=0.05(i=1,2, \ldots, 20)$. Further detailed geometric and physical parameters of the system are given in reference [18]. The power flow spectra are expressed in decible scale ( $\mathrm{dB}$ reference: $\left.10^{-12} \mathrm{~W}\right)$.

Figure 8 shows a comparison of the power flow in the hybrid control system to the passive one. It is observed that the hybrid isolation system provides a reduction in the total vibratory power transmitted to the plate. Compared to the simple hybrid isolation system presented in Section 2, we observe that for the multiple dimensional hybrid system the power flow spectra are of a very complex nature. There now exist many more resonance peaks in the power flow spectra curves which are associated with the natural modes of the flexible supporting foundation.

Figure 9 illustrates a comparison of the power flow spectra determined for various feedback gain values assumed in the hybrid control system. It can be concluded from this evidence that an increase of the control gain benefits in a reduction of the power flow transmission. Significant suppression of the peaks of the power flow spectra are observed especially in the low frequency range even for a small feedback gain.

The effect of the system's symmetry on power flow transmission is shown in Fig. 10. It can be seen that the symmetric arrangement of the whole system (i.e., symmetric load $F_{s}=\left\{0,0, e^{j \omega t}\right\}^{T}$, a three-dimensional geometrical structure and material property parameters) leads to a very good performance of the passive isolation system, which approximately reaches the same isolation efficiency as the hybrid control strategy assuming $K_{c}=50$. This figure provides guidelines to the desirable placement of mountings on flexible structures in a passive system. It is suggested, therefore, that isolators be mounted as symmetrically as possible thereby reducing vibration transmission without much active energy input. 


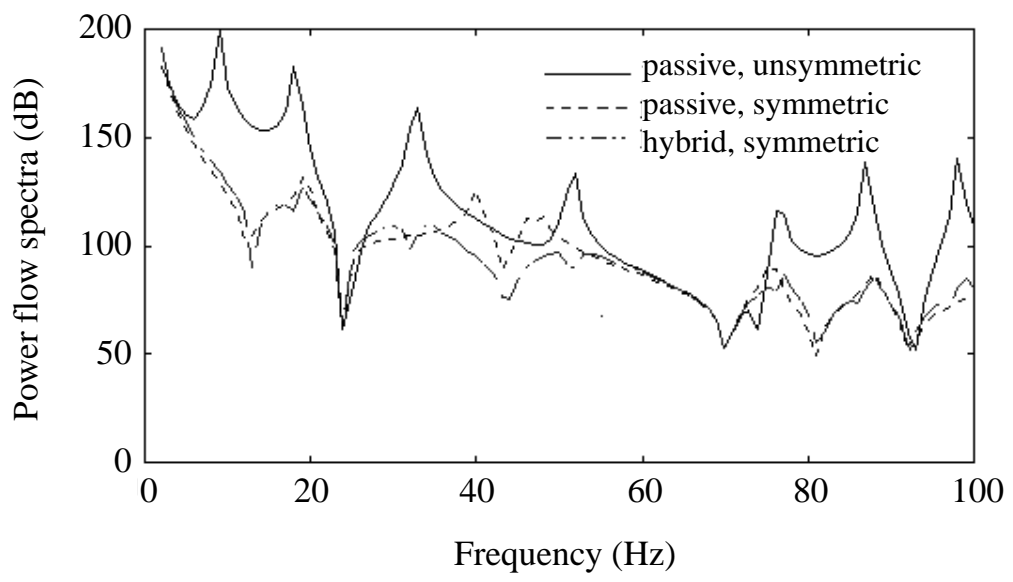

Fig. 10. Effect of symmetry of system on power flow transmission spectra.

\section{Conclusions}

A hybrid active and passive vibration control approach is developed treating the total power flow transmission as a performance index and a hybrid control mechanism demonstrated from the viewpoint of power flow. A mathematical model is presented describing the dynamic interactions between machine, passive/active controllers and the dynamics of the supporting structure for a multi-dimensional flexible coupled system subject to multiple excitations. The actuators, collocated with the passive isolators, governed by a proportional force feedback control law are successfully used to produce active force cancellation in the mounts creating a dynamic stiffness softening effect without diminishing the loading capability of the isolators. Hence, the hybrid active and passive isolation system possesses a much lower resonance frequency than the equivalent passive one. This therefore results in an extended frequency range with better vibration isolation effectiveness. The computational simulations for a simple and a more practical complex hybrid isolation systems subject to multiple excitations demonstrate that this hybrid power flow control method is very effective to reduce power flow transmissions from sources to receivers and to improve the overall performance of the isolation system over a wider range of frequencies. Of particular interest is the significant improved control capability provided by the symmetric placement of the mounts with respect to the foundation.

\section{Acknowledgement}

This research is partially supported by NSFC and NSF of Shandong Province. We express our deep ap- preciation to the Royal Society for supporting Professor Ye-Ping Xiong to do the related corroborative research in the UK.

\section{References}

[1] R.E.D. Bishop and D.C. Johnson, The Mechanics of Vibration, Cambridge University Press, 1960.

[2] J.I.Bushch-Vishniac, A fundamental problem with mobility analysis of vibration isolation systems, Journal of Acoustical Society of America 81(3) (1987), 1801-1804.

[3] M.A. Franchek, M.W. Ryan and R.J. Bernhard, Adaptive passive vibration control, Journal of Sound and Vibration 189(5) (1996), 565-585.

[4] H.G.D. Goyder and R.G. White, Vibrational power flow from machines into buildup structures, part III: power flow through isolation systems, Journal of Sound and Vibration 68(1) (1980), 97-117.

[5] K. Grigoriadis, G. Zhu and R. Skelton, Optimal redesign of linear systems, J. Dyn. Sys., Measurement and Control 118 (1996), 598-605.

[6] C.M. Harris, Shock and Vibration Handbook, McGraw Hill, New York, 1988.

[7] M.D. Jenkins, P.A. Nelson, R.J. Pinnington and S.J. Elliott, Active isolation of periodic machinery vibrations, Journal of Sound and Vibration 166(1) (1993), 117-140.

[8] M. Kawatani, Y.Yamada, M.Shimono and A.Mori, Hybrid control of traffic-induced vibration of girder bridges, Proc. First World Conf. on Struct. Control TA2 (1994), 3-10.

[9] D.J. Leo and D.J. Inman, A quadratic programming approach to the design of active-passive vibration isolation systems, Journal of Sound and Vibration 220(5) (1998), 807-825.

[10] T.E. Pare and J.P. How, Hybrid H-2 Control Design For Vibration Isolation, Journal of Sound and Vibration 226(1) (1999), 25-39.

[11] K.B. Scribner, L.A. Sievers and A.H. Von Flotow, Active narrow-band vibration isolation of machinery noise from resonant substructure, Journal of Sound and Vibration 167(1) (1993), 17-40.

[12] M. Sener and S. Utku, Adaptive base isolation system for the control of seismic energy flow into buildings, Journal of 
Intelligent Material Systems And Structures 9(2) (1998), 104115.

[13] P.B. Shing, M. Dixon, M. Kermiche and R. Su, Hybrid control techniques for building structures, Proc. First World Conf. on Struct. Control WP2 (1994), 100-109.

[14] J.C. Snowdon, Vibration isolation: use and characteristics, Journal of Acoustical Society of America 66 (1979), 12451279.

[15] Y.P. Xiong, K.J. Song and X. Ai, Influence of flexible foundation on isolator wave effects, Journal of Shock and Vibration 3(1) (1996), 64-70.
[16] Y.P. Xiong and K.J. Song, Power flow analysis for a new isolation system - flexible floating raft, Chinese Journal of Mechanical Engineering 9(3) (1996), 260-264.

[17] Y.P. Xiong and K.J. Song, Optimum control of vibration and structure-borne noise for machinery on flexible foundation, Chinese Journal of Mechanical Engineering 99(1) (1996), 1320.

[18] C.D. Yang, Power flow analysis for the coupling system of machine-isolators-plate subject to multiple excitations (in Chinese), Msc Thesis, Shandong University of Technology, 1998. 

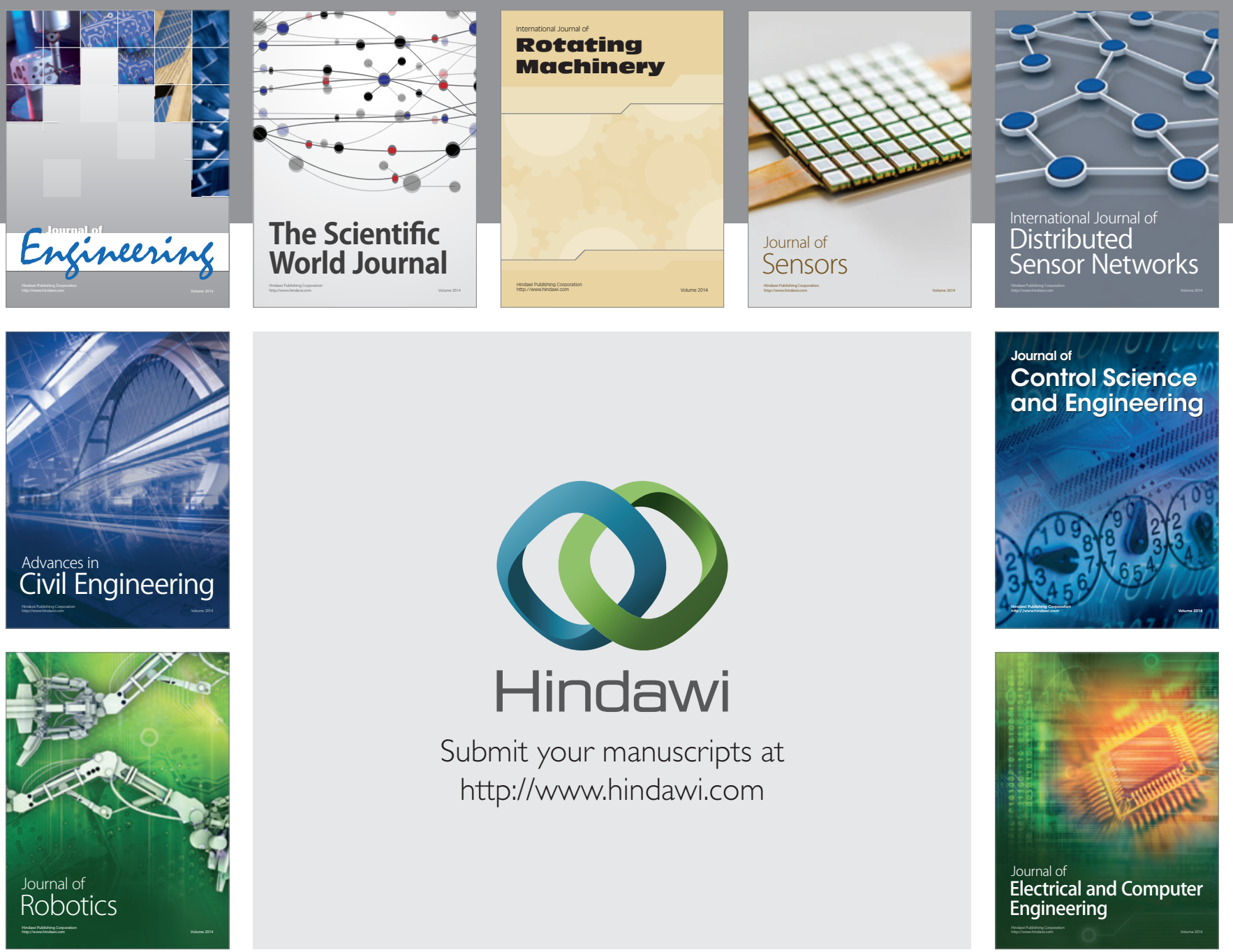

Submit your manuscripts at

http://www.hindawi.com
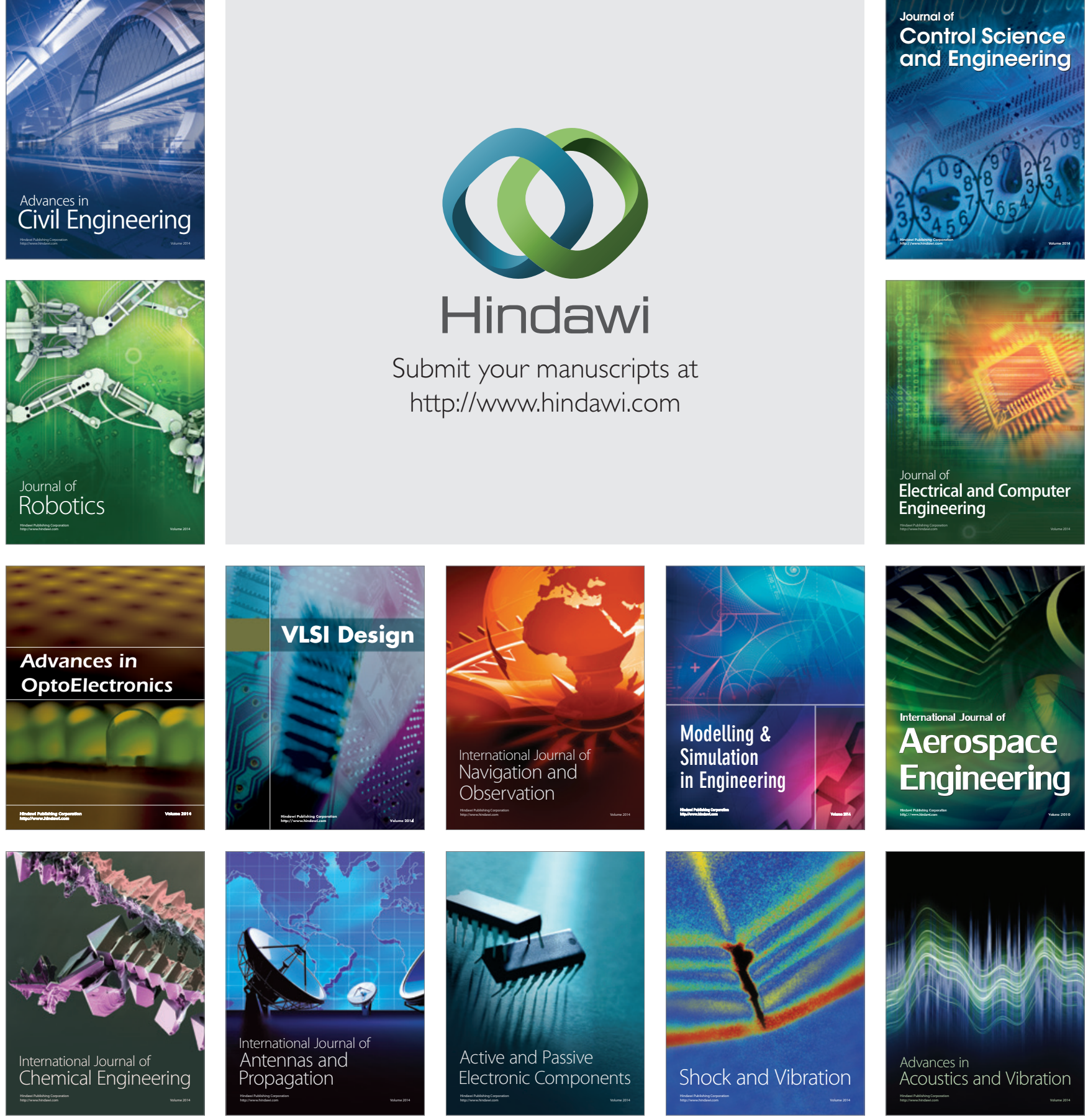